\title{
Short Communication Sex-specific risk factor profile in oesophageal adenocarcinoma
}

\author{
HE LöfdahI', I, Y Lu',2 and J Lagergren' \\ 'Unit of Esophageal and Gastric Research (ESOGAR), Department of Molecular Medicine and Surgery, Karolinska Institutet, Stockholm, Sweden; \\ ${ }^{2}$ Department of Medical Epidemiology and Biostatistics, Karolinska Institutet, Stockholm, Sweden
}

A nationwide Swedish case-control study of 388 men and 63 women with adenocarcinoma of the oesophagus and gastrooesophageal function and 676 men controls and 140 women investigated whether sex differences in aetiology contribute to male predominance. Compared with men, women seemed more vulnerable to reflux (odds ratio $(O R)=4.6,95 \%$ confidence interval $(\mathrm{Cl})=2.0-10.5$ vs $\mathrm{OR}=3.4,95 \% \mathrm{Cl}=2.5-4.6)$, obesity $(\mathrm{OR}=10.3,95 \% \mathrm{Cl}=2.6-42.3$ vs $\mathrm{OR}=5.4,95 \% \mathrm{Cl}=2.6-10.8)$ and smoking $(\mathrm{OR}=5.3,95 \% \mathrm{Cl}=2.0-\mid 4 . \mathrm{l}$ vs $\mathrm{OR}=2.8,95 \% \mathrm{Cl}=1.9-4.2)$, less harmed by low intake of fruit and vegetables $(\mathrm{OR}=0.9$, $95 \% \mathrm{Cl} 0.3-2.4$ vs $\mathrm{OR}=1.6,95 \% \mathrm{Cl}=1 . \mathrm{I}-2.2)$ and less protected by Helicobacter pylori infection $(\mathrm{OR}=0.5,95 \% \mathrm{Cl}=0.3-0.8 \mathrm{vs}$ $\mathrm{OR}=1.6,95 \% \mathrm{Cl}=0.5-5.4)$.

British Journal of Cancer (2008) 99, I506- 15 I0. doi:I0.1038/sj.bjc.660470 I www.bjcancer.com

Published online 7 October 2008

(c) 2008 Cancer Research UK

Keywords: gender; neoplasm; oesophagus; cardia; population based

The striking 6-8:1 male predominance of adenocarcinoma of the oesophagus, including the gastro-oesophageal junction (Vizcaino et al, 2002), has not yet been explained. An understanding of this gender difference might help explain why the incidence of this tumour is increasing more rapidly than that of any other malignancy in several populations (Vizcaino et al, 2002), and may help to identify preventive and therapeutic strategies. Three established risk factors are gastro-oesophageal reflux (Lagergren et al, 1999a; Shaheen and Ransohoff, 2002; Vakil et al, 2006), obesity (Lagergren et al, 1999b) and tobacco smoking (Lagergren et al, 2000), whereas the two protective factors are high intake of fruits and vegetables (Terry et al, 2001) and infection with Helicobacter pylori (H. pylori) (Ye et al, 2004; Rokkas et al, 2007). We hypothesised that male predominance in oesophageal adenocarcinoma is due atleast to established risk factors having a more harmful effect on men than women and that protective factors have a stronger preventive effect on women than men. To test this hypothesis, we conducted gender-specific analyses of the five established aetiologic factors in a population-based case-control study in Sweden.

\section{METHODS}

We have previously examined the five established aetiological factors in our Swedish population-based case-control study of adenocarcinoma of the oesophagus and gastro-oesophageal junction (Lagergren et al, 1999a, b, 2000; Terry et al, 2001; Ye et al, 2004), but gender-specific analyses or analyses combining both these sites have not been presented. The study design is described

*Correspondence: HE Löfdahl, Unit of Esophageal and Gastric Research (ESOGAR), P9:03, Department of Molecular Medicine and Surgery, Karolinska Institutet, Stockholm SE-17I 76, Sweden;

E-mail: hedvig-elisabet.lofdahl.348@student.ki.se

Received 21 July 2008; revised 5 September 2008; accepted 10 September 2008; published online 7 October 2008 elsewhere (Lagergren et al, 1999a). Briefly, it is based on the entire Swedish-born population, living in Sweden during 1995-97, below 80 years of age. Cases were identified shortly after diagnosis through collaboration among all 195 relevant hospital departments and all six tumour registers, which reduced non-participation due to weakness or early mortality. To decrease misclassification, special routines for documentation and prospective reporting of tumour site and type were used by endoscopists, surgeons and pathologists. The tumour had to be adenocarcinoma in type and to have its centre within the oesophagus or a maximum $3 \mathrm{~cm}$ distal to the gastro-oesophageal junction. The tumour specimens were finally re-examined by one pathologist. Control participants were randomly selected from the population register and frequency-matched to cases for age and sex. Participants were interviewed face-to-face about exposures and background data by professional interviewers who were unaware of the study hypotheses and trained to deal with the cases and controls in exactly the same manner. Regarding reflux, questions were asked about recurrent heartburn and regurgitation, that is, the symptoms on which a diagnosis of gastro-oesophageal reflux is based (Vakil et al, 2006). To avoid tumour influence on the reported exposure, we disregarded reflux symptoms less than 5 years before the interview. To assess the effects of body mass index (BMI), participants were asked about their weight and height 20 years before the interview, their maximum and minimum weight as adults, and their weight at the age of 20 years (Lagergren et al, 1999b). Concerning tobacco smoking, the user status was recorded as any type of tobacco use 2 years before the interview, whereas frequency and duration of tobacco smoking was based on cigarette smoking alone (Lagergren et al, 2000). The total average consumption of all fruits and vegetables 20 years before the interview was assessed from a food frequency questionnaire (Terry et al, 2001). The participants were asked to provide a venous blood sample for $H$. pylori testing. Serum immunoglobulin $\mathrm{G}$ antibodies against $H$. pylori and its virulence factor Cag A were measured by immunosorbent assay (ELISA) as described earlier (Ye et al, 2004).

Unconditional logistic regression was used to estimate odds ratios (ORs) with 95\% confidence intervals (CIs) for all study 
exposures in sex-stratified analyses. In multivariable models, the ORs were adjusted for age, educational level, alcohol use, reflux, BMI, tobacco smoking, intake of fruits and vegetables, and H. pylori infection. The categorisation of each of these included variables in the model is presented in Tables 1 and 2.

\section{RESULTS}

Among 529 eligible cases and 1128 eligible controls, 451 (85\%) and $816(72 \%)$, respectively, participated in the interview. Among the

Table I Characteristics of female and male cases and controls

\begin{tabular}{|c|c|c|c|c|}
\hline \multirow[b]{2}{*}{ Variables } & \multicolumn{2}{|c|}{ Women } & \multicolumn{2}{|c|}{ Men } \\
\hline & $\begin{array}{l}\text { Cases } \\
N(\%)\end{array}$ & $\begin{array}{c}\text { Controls } \\
N(\%)\end{array}$ & $\begin{array}{l}\text { Cases } \\
N(\%)\end{array}$ & $\begin{array}{c}\text { Controls } \\
N \text { (\%) }\end{array}$ \\
\hline Total & 63 & 140 & 388 & 676 \\
\hline \multicolumn{5}{|c|}{ Age group (years at interview) } \\
\hline $0-49$ & $5(7.9)$ & $10(7.1)$ & $28(7.2)$ & $38(5.6)$ \\
\hline $50-59$ & $9(14.3)$ & $24(17.1)$ & $71(18.3)$ & $136(20.0)$ \\
\hline $60-69$ & $14(22.2)$ & $39(27.9)$ & $131(33.8)$ & $206(30.5)$ \\
\hline $70-79$ & $35(55.6)$ & $67(47.9)$ & $158(40.7)$ & $296(43.8)$ \\
\hline \multicolumn{5}{|c|}{ Formal education (years) } \\
\hline $0-9$ & $45(71.4)$ & $74(52.9)$ & $266(68.6)$ & $423(62.6)$ \\
\hline $10-12$ & $12(19.1)$ & $33(23.6)$ & $68(17.5)$ & $126(18.6)$ \\
\hline$>13$ & $6(9.5)$ & $33(23.6)$ & $54(13.9)$ & $127(18.8)$ \\
\hline \multicolumn{5}{|c|}{ Alcohol status (current usage 20 years before interview) } \\
\hline No & $20(31.8)$ & $39(27.9)$ & $55(14.2)$ & $92(13.6)$ \\
\hline Yes & $43(68.3)$ & $101(72.1)$ & $333(85.8)$ & $584(86.4)$ \\
\hline
\end{tabular}

cases, 63 were women (14\%), with a male-to-female ratio of $7: 1$. Some characteristics of the study participants are presented in Table 1. Most patients were aged 70-79 years. Female cases had on average, a shorter education than controls and male cases and controls. Use of alcohol was higher among men, but similar between cases and controls in each sex. Table 2 presents an overview of the sex-stratified risk estimates of the five studied aetiological factors in relation to risk of oesophageal and junctional adenocarcinoma. Reflux symptoms showed a slightly higher risk estimate in women (OR 4.6, 95\% CI $2.0-10.5)$ than in men (OR 3.4, 95\% CI 2.5-4.6) at least weekly. An increased severity and duration of reflux was associated with higher risk estimates among women than among men (Table 3 ).

Women with a long history of reflux ( $>20$ years) had an OR of 12.3 (95\% CI 2.9-51.4), whereas the corresponding OR in men was 6.6 (95\% CI 3.8-11.6). A high BMI was associated with higher point estimates in women than in men (Tables 2 and 4); obese women had an OR of 10.3 (95\% CI 2.6-42.3) and obese men of 5.4 (95\% CI 2.6-10.8). Also, a greater increase in BMI between the lowest and highest adult BMI rendered a higher OR among women than among men (Table 4). Current tobacco smoking was associated with a higher risk estimate in women (OR 5.3, 95\% CI $2.0-14.1$ ) than in men (OR 2.8, 95\% CI 1.9-4.2), and a higher frequency of cigarette smoking ( $>19$ per day) showed a higher risk in women (OR 5.7, 95\% CI 1.3-25.6) than in men (OR 2.3, 95\% CI 1.6-3.4) (Table 5). An increased risk associated with low intake of fruits and vegetables was found only in men (men OR 1.6, 95\% CI 1.1-2.2; women 0.9 , 95\% CI $0.3-2.4$ ) (Table 2). The inverse risk among $H$. pylori-infected men (OR 0.5 , 95\% CI $0.3-0.8$ ) was not seen in women (OR 1.6, 95\% CI 0.5-5.4) (Table 2). The point estimates from separate analyses of oesophageal and gastro-oesophageal junctional adenocarcinoma did not reveal any material male-female differences (data not shown).

Table 2 Risk factor profile, women vs men, for oesophageal and cardia adenocarcinoma, expressed in OR with 95\% C

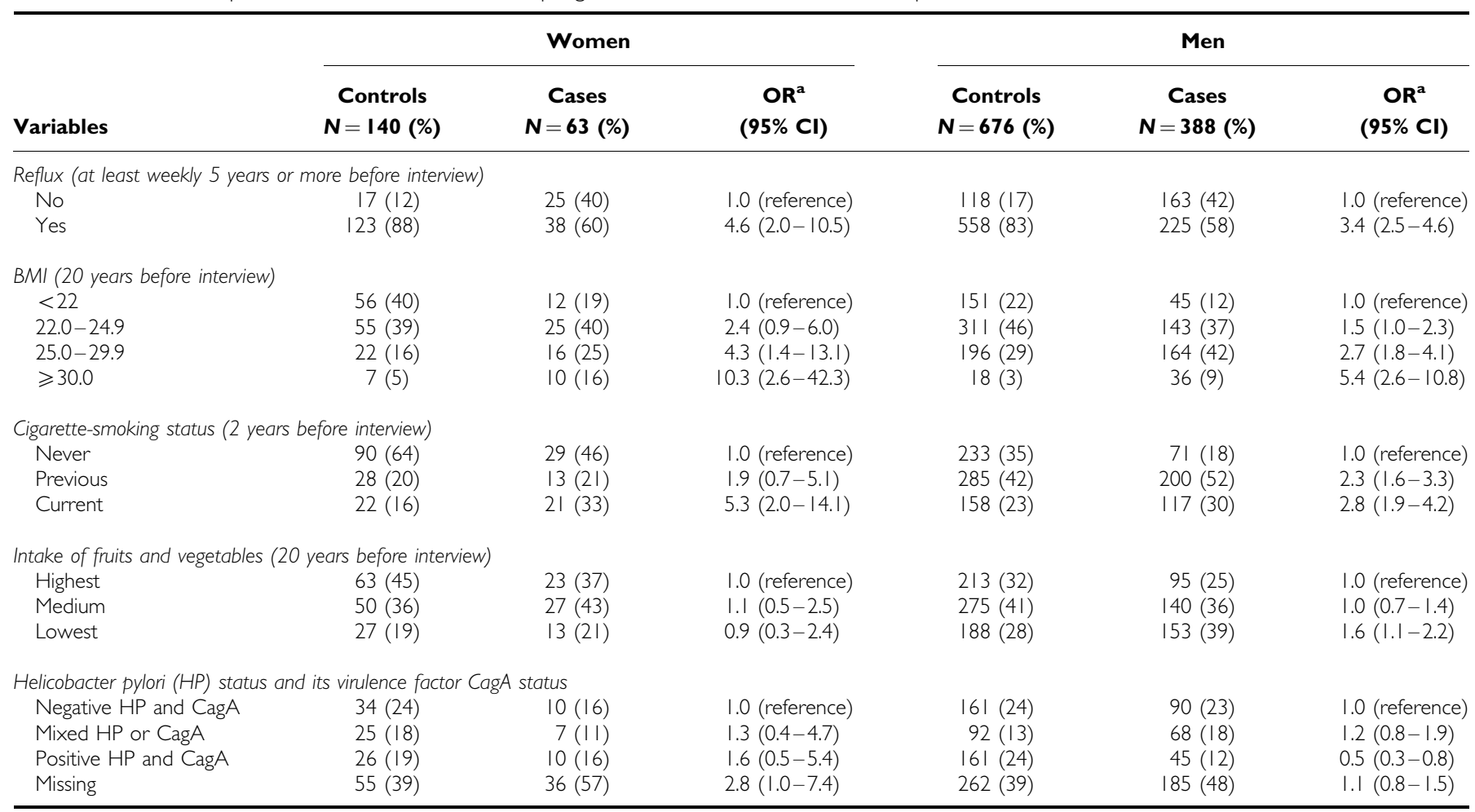

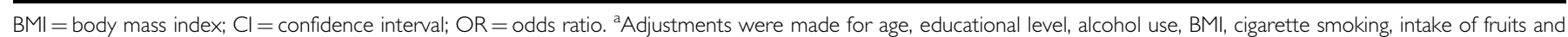
vegetables, and Helicobacter pylori infection. 
Table 3 Gastro-oesophageal reflux symptoms and risk of oesophageal and cardia adenocarcinoma expressed in OR with $95 \% \mathrm{Cl}$

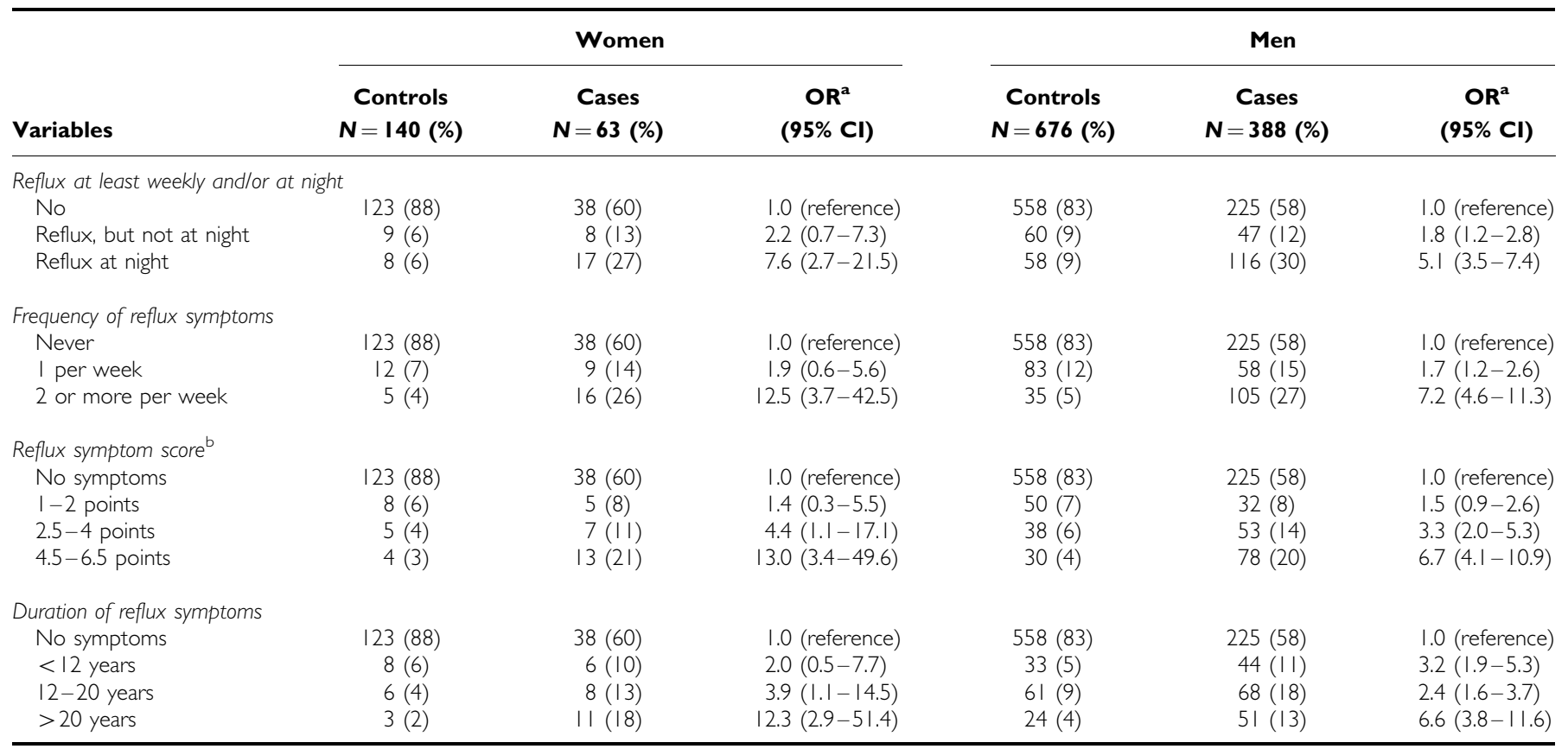

$\mathrm{BMI}=$ body mass index; $\mathrm{Cl}=$ confidence interval; $\mathrm{OR}=$ odds ratio. ${ }^{2}$ Adjustments were made for age, educational level, alcohol use, BMl, cigarette smoking, intake of fruits and vegetables, and Helicobacter pylori infection. ${ }^{\text {b}}$ The index score included symptom characteristics (heartburn only $=\mid$ point, regurgitation only $=\mid$ point, heartburn and regurgitation combined $=1.5$ points), nightly symptoms (no $=0$ point, yes $=2$ points) and symptom frequency (once a week $=0$ point, $2-6$ times a week $=1$ point, $7-15$ times a week $=2$ points, $>15$ times a week $=3$ points).

Table $4 \mathrm{BMl}$ and risk of oesophageal and cardia adenocarcinoma expressed in OR with $95 \% \mathrm{Cl}$

\begin{tabular}{|c|c|c|c|c|c|c|}
\hline Variables & \multicolumn{3}{|c|}{ Women } & \multicolumn{3}{|c|}{ Men } \\
\hline \multicolumn{7}{|c|}{ BMI maximum as adult } \\
\hline$<22$ & $18(13)$ & $2(3)$ & 1.0 (reference) & $40(6)$ & $12(3)$ & 1.0 (reference) \\
\hline $22-24.9$ & $44(31)$ & $11(17)$ & I.I $(0.2-6.3)$ & $198(29)$ & 77 (20) & I.I $(0.5-2.4)$ \\
\hline $25-29.9$ & $56(40)$ & $26(4 I)$ & $3.1(0.6-16.9)$ & $335(50)$ & $208(54)$ & $1.8(0.9-3.6)$ \\
\hline \multicolumn{7}{|c|}{ BMI minimum as adult } \\
\hline$<22$ & $112(80)$ & $32(5 \mathrm{I})$ & I.0 (reference) & $352(52)$ & $147(38)$ & 1.0 (reference) \\
\hline $22-24.9$ & $23(16)$ & $24(38)$ & $4.1(1.7-9.6)$ & $270(40)$ & $183(47)$ & $1.6(1.2-2.2)$ \\
\hline$\geqslant 25$ & $5(4)$ & $7(12)$ & $7.6(1.9-30.5)$ & $54(8)$ & $58(15)$ & $2.7(1.7-4.2)$ \\
\hline \multicolumn{7}{|c|}{ BMI change during adult life } \\
\hline$>2$ & $15(\mathrm{I} \mid)$ & $4(6)$ & I.0 (reference) & $121(18)$ & $59(15)$ & 1.0 (reference) \\
\hline
\end{tabular}

\section{DISCUSSION}

This study finds, in contradiction to our hypothesis, that exposures to reflux, obesity and tobacco are stronger risk factors for oesophageal adenocarcinoma in women than in men and that the protective effects of fruits and vegetables and infection with $H$. pylori observed in men do not occur in women.

Study strengths include the nationwide and population-based design with high participation, face-to-face interviews, detailed exposure information and a thorough tumour classification.
Although we included virtually all female cases in the whole of Sweden during a 3-year period, to offset the low incidence of oesophageal adenocarcinoma in women, we combined it with adenocarcinoma of gastro-oesophageal junction, as justified by their similar sex distributions and risk factor profiles (Vizcaino et al, 2002).

During the last few years, it has been established that reflux and obesity (Lagergren et al, 1999a, b; Shaheen and Ransohoff, 2002; Vakil et al, 2006) are independent and strong risk factors for oesophageal adenocarcinoma, whereas tobacco smoking is a 
Cigarette smoking and risk of oesophageal and cardia adenocarcinoma expressed in OR with $95 \% \mathrm{Cl}$

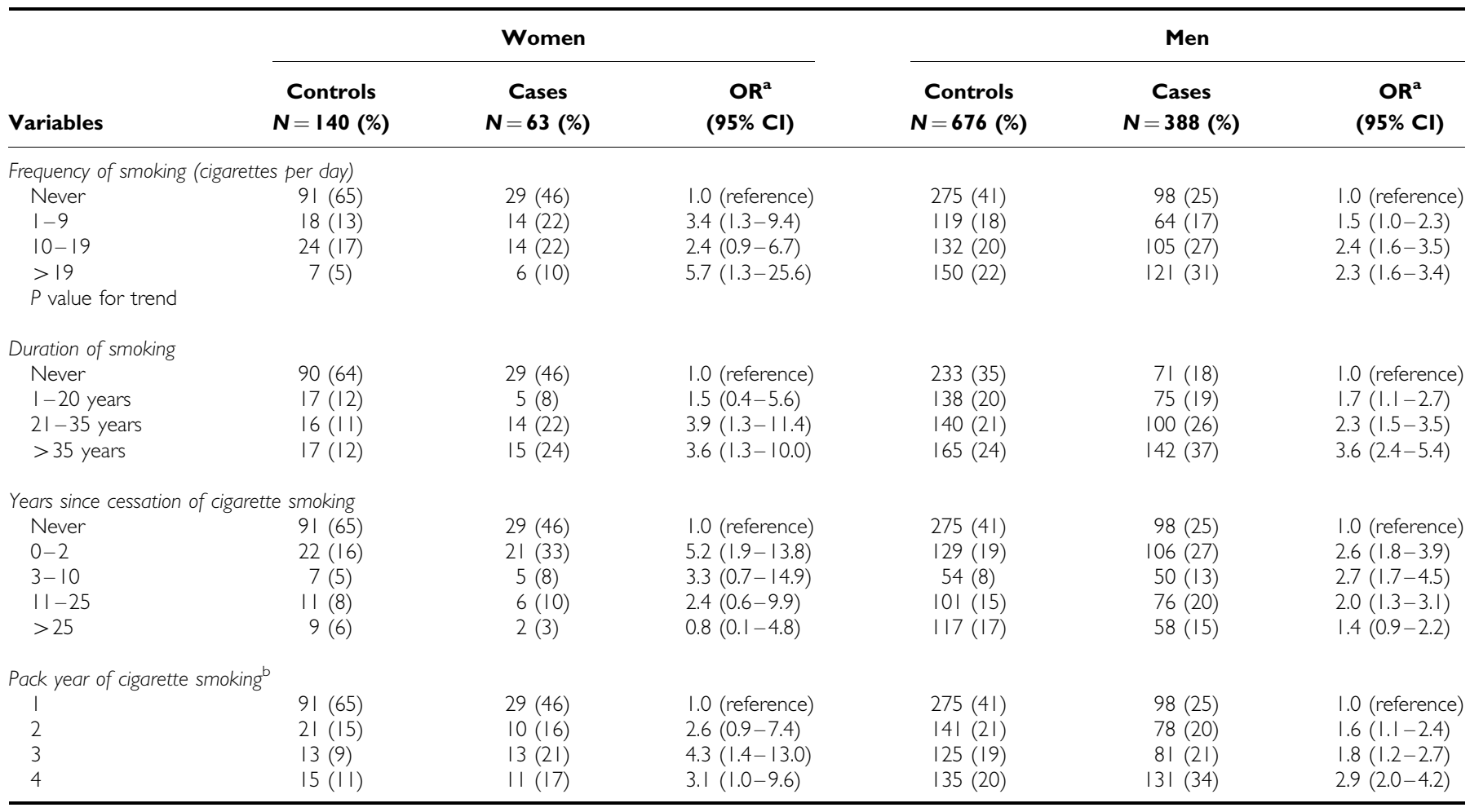

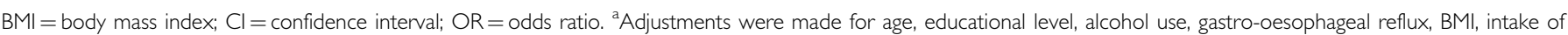
fruits and vegetables, and Helicobacter pylori infection. ${ }^{b}$ Number of cigarettes per day times the total years of smoking.

moderate risk factor (Lagergren et al, 2000). Dietary intake of fruits and vegetables (Terry et al, 2001) and infection with $H$. pylori (Ye et al, 2004; Rokkas et al, 2007) are inversely associated with risk of this cancer. All these results are entirely in agreement with the findings in our case-control study (Lagergren et al, 1999a,b, 2000; Terry et al, 2001; Ye et al, 2004), indicating good validity. As however, a striking majority of the patients are men and most studies have not analysed women separately, available work has established aetiologic factors only among men. This study, undertaken mainly to compare risk factor profiles among women and men using a large data source of high validity, finds that women do not seem less vulnerable to risk exposures or more protected by preventive factors than men, but rather suggests the opposite. A possible explanation might be that women are exclusively protected from developing oesophageal adenocarcinoma by some yet unidentified factors, for example, female sex hormones. A protective role of oestrogen has been evaluated in some studies, but with no clear evidence of the effect, although the statistical power was limited (Lagergren and Jansson, 2005;

\section{REFERENCES}

Chandanos E, Lindblad M, Jia C, Rubio CA, Ye W, Lagergren J (2006) Tamoxifen exposure and risk of oesophageal and gastric adenocarcinoma: a population-based cohort study of breast cancer patients in Sweden. Br J Cancer 95: 118 - 122

Cheng KK, Sharp L, McKinney PA, Logan RFA, Chilvers CED, CookMozaffari P, Ahmed A, Day NE (2000) A case-control study of oesophageal adenocarcinoma in women: a preventable disease. $\mathrm{Br} J$ Cancer 83: $127-132$

Lagergren J, Bergstrom R, Lindgren A, Nyrén O (1999a) Symptomatic gastroesophageal reflux as a risk factor for esophageal adenocarcinoma. $N$ Engl J Med 340: 825-831
Chandanos et al, 2006). However, a UK case-control study noted a decreased risk among women with a history of breast feeding (Cheng et al, 2000).

This study suggests that with regard to oesophageal adenocarcinoma risk, women are as vulnerable as men to exposure to reflux, obesity and tobacco, whereas the protective effects of fruits and vegetables and infection with $H$. pylori might be confined to men. Thus, the sex difference in oesophageal adenocarcinoma does not seem to be explained by differences in risk factor profiles of known aetiological agents.

\section{ACKNOWLEDGEMENTS}

We are grateful to all of the 227 doctors who acted as contact persons at the participating departments and provided invaluable input during the planning of the study. Funding was provided by the National Cancer Institute (NCI) grant R01 CA57947-03, the Swedish Cancer Society and Swedish Research Council (JL).

Lagergren J, Bergstrom R, Nyren O (1999b) Association between body mass and adenocarcinoma of the esophagus and gastric cardia. Ann Intern Med 130: 883-890

Lagergren J, Berström R, Lindgren A, Nyrén O (2000) The role of tobacco, snuff and alcohol use in the aetiology of cancer of the oesophagus and gastric cardia. Int J Cancer 85: 340-346

Lagergren J, Jansson C (2005) Sex hormones and oesophageal adenocarcinoma: influence of childbearing? Br J Cancer 93: 859-856

Rokkas T, Pistiolas D, Sechopoulos P, Robotis I, Margantinis G (2007) Relationship between Helicobacter pylori infection and esophageal neoplasia: a meta-analysis. Clin Gastroenterol Hepatol 5: 1413-1417 
Shaheen N, Ransohoff DF (2002) Gastroesophageal reflux, barrett esophagus, and esophageal cancer: scientific review. JAMA 287: $1972-1981$

Terry P, Lagergren J, Hansen H, Wolk A, Nyrén O (2001) Fruit and vegetable consumption in the prevention of oesophageal and cardia cancers. Eur J Cancer Prev 10: 365-369

Vakil N, van Zanten SV, Kahrilas P, Dent J, Jones R, Global Consensus Group (2006) Global Consensus Group. The Montreal definition and classification of gastroesophageal reflux disease: a global evidence-based consensus. Am J Gastroenterol 101: $1900-1920$
Vizcaino AP, Moreno V, Lambert R, Parkin DM (2002) Time trends incidence of both major histologic types of esophageal carcinomas in selected countries, 1973-1995. Int J Cancer 99: $860-868$

Ye W, Held J, Lagergren J, Engstrand L, Blot WJ, McLaughlin JK, Nyrén O (2004) Helicobacter pylori infection and gastric atrophy: risk of adenocarcinoma and squamous-cell carcinoma of the esophagus and adenocarcinoma of the gastric cardia. J Natl Cancer Inst 96(5): $388-396$ 